\title{
On The Determination of Shadow Boundaries and Relevant Eigenrays For Sound Propagation In Stratified Moving Media
}

\author{
Gerry L. Mcaninch ${ }^{1}$ and Stephanie L. Heath ${ }^{2}$ \\ NASA Langley Research Center, Hampton Virginia 23682-2199
}

The eiconal and ray equations for a stratified moving medium are analyzed to determine which rays connect a source located at an altitude $\mathrm{z}=\mathrm{z}_{\mathrm{s}}$ to an observer on the ground plane. The characteristics of all available rays are ascertained. Further, the rays which determine the shadow boundary are found. Thus, the shadow boundary may be easily calculated and any observers for which an eigenray does not exist eliminated from further consideration. Therefore, the analysis may be applied to save computer time in two ways. First, rays that do not reach the ground may be eliminated before they are traced, and observers for which no eigenray exists may be eliminated from consideration.

\section{Introduction}

The geometric acoustic theory ${ }^{1-5}$ provides a very flexible method for the study of sound propagation in the earth's atmosphere. However, in the geometric theory the determination of the acoustic field at a fixed observer location requires the solution of a two-point boundary value problem for the eigenray. If the medium through which the disturbance propagates is assumed to be stationary and homogeneous, this problem has a very simple geometric solution - so simple, in fact, that we solve it without realizing that a problem has arisen - and very little computation time is required to determine the eigenray and the geometric spreading losses. However, if the sound speed is assumed to vary, perhaps with altitude alone as would be fitting for atmospheric propagation studies, two problems arise which are absent in the stationary, homogeneous medium problem. First, a shadow boundary, which divides the plane on which the observers reside into an ensonified region and a zone of silence, or shadow zone, may exist. If such a shadow region exists, there are no rays connecting the source to observers in that region. Thus, any computer time spent attempting to find such a ray is wasted. Second, some rays that would reach the observer plane in the stationary, homogeneous medium case, fail to do so when the sound speed varies with altitude, while some rays that would not reach the ground in the stationary, homogeneous medium case, may do so when the sound speed varies with altitude. Clearly, tracing rays that do not reach the observer plane wastes computer time, while not considering rays that do leads to erroneous noise predictions. If only the stationary inhomogeneous medium case is of interest, simple well known criteria

${ }^{1}$ Senior Aeronautical Engineer, Structural Acoustics Branch, MS 463 NASA LaRC.

${ }^{2}$ Aeronautical Engineer, Aeroacoustics Branch, MS 461 NASA LaRC, Member AIAA. 
can be applied to overcome these problems ${ }^{6-7}$. If, however, the effects of a sheared mean flow are important, the required criteria are neither so simple, nor as well known.

In the following, analysis of the eiconal and ray equations will provide a complete description of the behavior of any ray before that ray is traced, allowing rays that do not reach the ground to be eliminated from consideration. Further, the set of rays that make up the shadow boundary is determined. Thus, the tracing of a relatively limited number of rays allows the determination of the shadow boundary and the elimination of observers that cannot be reached by any ray.

\section{Notation and Fundamental Equations}

In the following the main interest is determining which rays will carry energy from a source at an altitude $z_{s}$ to an observer at $z=0$. The source is assumed to be embedded in a medium whose sound speed $\mathrm{c}(\mathrm{z})$ and mean flow velocity $\stackrel{\mathrm{v}}{\mathrm{U}}(\mathrm{z})=\mathrm{U}(\mathrm{z}) \hat{\mathrm{x}}+\mathrm{V}(\mathrm{z}) \hat{\mathrm{y}}=\mid \stackrel{\mathrm{v}}{\mathrm{v}}[\hat{\mathrm{x}} \operatorname{Cos}(\beta)+\hat{\mathrm{y}} \operatorname{Sin}(\beta)]$ are functions of $\mathrm{z}$ alone. Here, and in the following, a caret over a letter indicates a unit vector. Thus, for example, the notation $\hat{x}$ indicates a unit vector directed in the positive $\mathrm{x}$ direction. Similarly, an arrow over a quantity indicates a vector that need not be a vector of unit length. In the following, consideration will be limited to obtaining useful information from the eiconal equation $^{4}$

$$
\mathrm{s}_{\mathrm{x}}^{2}+\mathrm{s}_{\mathrm{y}}^{2}+\mathrm{s}_{\mathrm{z}}^{2}=\frac{\left[1-\left(U \mathrm{~s}_{\mathrm{x}}+V \mathrm{~s}_{\mathrm{y}}\right)\right]^{2}}{\mathrm{c}^{2}}
$$

and the ray equations

$$
\frac{d \mathrm{x}_{\mathrm{r}}}{\mathrm{dt}}=\mathrm{c \hat {s }}+\stackrel{\mathrm{v}}{\mathrm{U}}
$$

Note that the vector

$$
\hat{s}=\frac{s_{x} \hat{x}+s_{y} \hat{y}+s_{z} \hat{z}}{\sqrt{s_{z}^{2}+s_{y}^{2}+s_{z}^{2}}}
$$

is a unit vector that is normal to the wave-front, or surface of constant phase, and that equation (2) simply states that the surface of constant phase moves normal to itself at the local sound speed c while being carried along at the local flow velocity by the mean flow. The three-dimensional curve $\stackrel{\mathrm{x}}{=} \mathrm{x}_{\mathrm{r}}$ obtained by integrating equation (2) is the path followed by a point on the constant phase surface as the disturbance propagates. It is, therefore, a ray. Employing equation (1), equation (3) may be written in the alternate form 


$$
\hat{\mathrm{s}}=\frac{\mathrm{cs}}{\left[1-\left(\mathrm{Us}_{\mathrm{x}}+\mathrm{Vs}_{\mathrm{y}}\right)\right]}
$$

For the stratified medium to which we restrict our attention, the quantities $s_{x}$ and $s_{y}$ are constant along any given ray ${ }^{4}$, and, given $s_{x}$ and $s_{y}, s_{z}$ is determined algebraically by solving equation (1). Hence, the rays are a two parameter $\left(s_{x}, s_{y}\right)$ family of curves in three dimensional space, and the following analysis seeks to limit the range of $s_{x}$ and $s_{y}$ such that any pair $\left(\mathrm{s}_{\mathrm{x}}, \mathrm{s}_{\mathrm{y}}\right)$ in the restricted range will provide a ray that connects a source on the plane $\mathrm{z}=\mathrm{z}_{\mathrm{s}}$ to an observer on the plane $\mathrm{z}=0$.

\section{The Eiconal Equation as an Equation for a Prolate Spheroid.}

An alternate form of equation (1),

$$
\frac{\left\{\frac{M_{x} s_{x}+M_{y} s_{y}}{M}-\left[\frac{-M}{c\left(1-M^{2}\right)}\right]\right\}^{2}}{\left[\frac{1}{\left(1-M^{2}\right) c}\right]^{2}}+\frac{\left[\frac{M_{x} s_{y}-M_{y} s_{x}}{M}\right]^{2}}{\left[\frac{1}{\sqrt{\left(1-M^{2}\right) c}}\right]^{2}}+\frac{s_{z}^{2}}{\left[\frac{1}{\sqrt{\left(1-M^{2}\right) c}}\right]^{2}}=1
$$

of value for some of the discussion to follow, is obtained by introduction of the notation

$$
\begin{aligned}
& \stackrel{\mathrm{v}}{\mathrm{M}}=\frac{\dot{U}}{\mathrm{c}}=\mathrm{M}_{\mathrm{x}} \hat{\mathrm{x}}+\mathrm{M}_{\mathrm{y}} \hat{\mathrm{y}} \\
& \mathrm{M}=\sqrt{\mathrm{M}_{\mathrm{x}}^{2}+\mathrm{M}_{\mathrm{y}}^{2}}
\end{aligned}
$$

and minor algebraic manipulation. Note that equation (5) is the equation of a prolate spheroid.

\section{$\underline{\text { Turning Points }}$}

At a turning point the ray is tangent to the plane $\mathrm{z}=\mathrm{z}_{\mathrm{t}}$. Propagation of the disturbance in the $z$ direction is reversed at this point, and it follows from equation (2) that $s_{z}=0$. This paper essentially reduces to a discussion of the turning points, i.e., those values of $s_{x}$ and $\mathrm{s}_{\mathrm{y}}$ that satisfy the equation

$$
\mathrm{s}_{\mathrm{x}}^{2}+\mathrm{s}_{\mathrm{y}}^{2}=\frac{\left[1-\left(\mathrm{U}_{\mathrm{t}} \mathrm{s}_{\mathrm{x}}+\mathrm{V}_{\mathrm{t}} \mathrm{s}_{\mathrm{y}}\right)\right]^{2}}{\mathrm{c}_{\mathrm{t}}^{2}}
$$


obtained by setting $\mathrm{s}_{\mathrm{z}}=0$ in equation (1). Here, the subscript $t$ implies that the subscripted quantity is evaluated at the turning point. Of particular interest, since they form the shadow boundary, are the turning points located at the ground. Since the mean flow is assumed to be zero at the ground, equation (8) becomes

$$
\mathrm{s}_{\mathrm{x}}^{2}+\mathrm{s}_{\mathrm{y}}^{2}=\frac{1}{\mathrm{c}_{0}^{2}}
$$

for this particular single parameter set of turning points. Here and in the following $\mathrm{c}_{0}$ is the sound speed at the ground.

\section{A Set of Significant Ellipses.}

Since equation (5) is the equation of a prolate spheroid, the equation

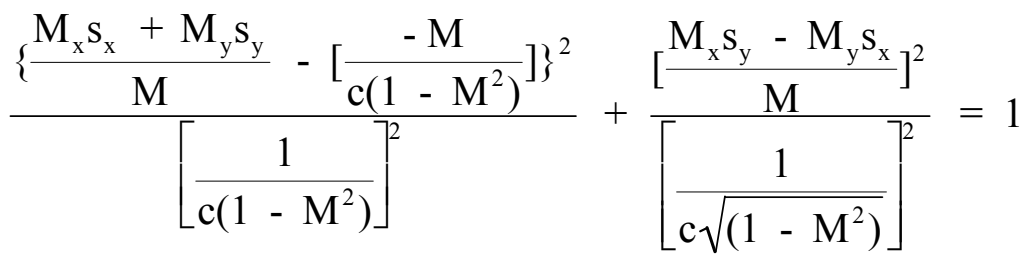

obtained from it by setting $\mathrm{s}_{\mathrm{z}}=0$, is the equation of an ellipse. That is, all rays that have a turning point at a given altitude $\mathrm{z}$ possess $\left(\mathrm{s}_{\mathrm{x}}, \mathrm{s}_{\mathrm{y}}\right)$ values that fall on this ellipse, referred to here as the ellipse of turning points (ETP). Note that both a coordinate rotation and a coordinate translation are required to place the coordinate origin at the center of the ellipse and to align the coordinate axis with the principal axis of the ellipse. The rotation is through the angle $\beta=\operatorname{Arctan}\left(\frac{\mathrm{V}}{\mathrm{U}}\right)$. Thus, the angle of the major axis of the ellipse to the $s_{x}$ axis is equal to the angle of the mean flow vector to the $x$-axis. The location of the center and the foci of the ellipse, and the eccentricity of the ellipse, easily determined because equation (10) is in standard form, are presented in the following Table. Further, it should be noted that for analytic functions $M_{x}(z), M_{y}(z)$, and $c(z)$, the motion of the ellipse is continuous as $\mathrm{z}$ changes.

Table. Properties of the Ellipse

\begin{tabular}{|l|l|}
\hline Center of ellipse & $\mathrm{s}_{\mathrm{x}}=\left[\frac{-\mathrm{MCos}(\beta)}{\mathrm{c}\left(1-\mathrm{M}^{2}\right)}\right] ; \mathrm{s}_{\mathrm{y}}=\left[\frac{-\mathrm{MSin}(\beta)}{\mathrm{c}\left(1-\mathrm{M}^{2}\right)}\right]$ \\
\hline Foci & $\left(\mathrm{s}_{\mathrm{x}}, \mathrm{s}_{\mathrm{y}}\right)=(0,0) ;$ \\
& $\left(\mathrm{s}_{\mathrm{x}}, \mathrm{s}_{\mathrm{y}}\right)=\left[\frac{-2 \mathrm{MCos}(\beta)}{\mathrm{c}\left(1-\mathrm{M}^{2}\right)}, \frac{-2 \mathrm{MSin}(\beta)}{\mathrm{c}\left(1-\mathrm{M}^{2}\right)}\right]$ \\
\hline Eccentricity & $\mathrm{M}$ \\
\hline
\end{tabular}


In order to visualize the variation of the ellipse with altitude, consider an atmosphere within which the wind speed, $\mathrm{U}^{(\mathrm{z})}=\mathrm{U}(\mathrm{z}) \hat{\mathrm{x}}$, increases monotonically with altitude, and for which the temperature, hence the sound speed, is constant. Then, the Mach number of the atmosphere will increase with altitude, and the set of curves generated either by equation (8) or, equivalently, equation (10) will consist of a circle centered at $\left(\mathrm{s}_{\mathrm{x}}, \mathrm{s}_{\mathrm{y}}\right)=$ $(0,0)$, obtained for $\mathrm{z}=0$, and ellipses with a common focus at $\left(\mathrm{s}_{\mathrm{x}}, \mathrm{s}_{\mathrm{y}}\right)=(0,0)$. The second focus which, for $\mathrm{z}>0$ separates from the first, moves in the negative $s_{x}$ direction along the $s_{x}$ axis. The distance of this second focus from the first increases with increasing Mach number, hence altitude, and, to first order in the Mach number, this distance is directly proportional to the Mach number, see the Table, Row 2. Also, see Figure 1, which shows three of these ellipses for an atmosphere with a flow speed increasing at $0.024(\mathrm{ft} / \mathrm{sec}) / \mathrm{ft}$. The first ellipse is for $\mathrm{z}=0$, the second for $\mathrm{z}=2,500 \mathrm{ft}$., and the third for $\mathrm{z}=10,000 \mathrm{ft}$. The motion of the center of the ellipse, and the second focus of the ellipse, along the negative $\mathrm{s}_{\mathrm{x}}$-axis is clearly indicated. Note that each ellipse has an eccentricity equal to the Mach number. Since $M<<1$, each ellipse is nearly a circle with a radius $r=1 / c$. Thus, if the temperature, hence the sound speed, decreases with altitude, as is normally the case, the radius of the approximating circle will increase with altitude. If, however, the temperature increases with altitude, the radius of the approximating circle will decrease with altitude. 


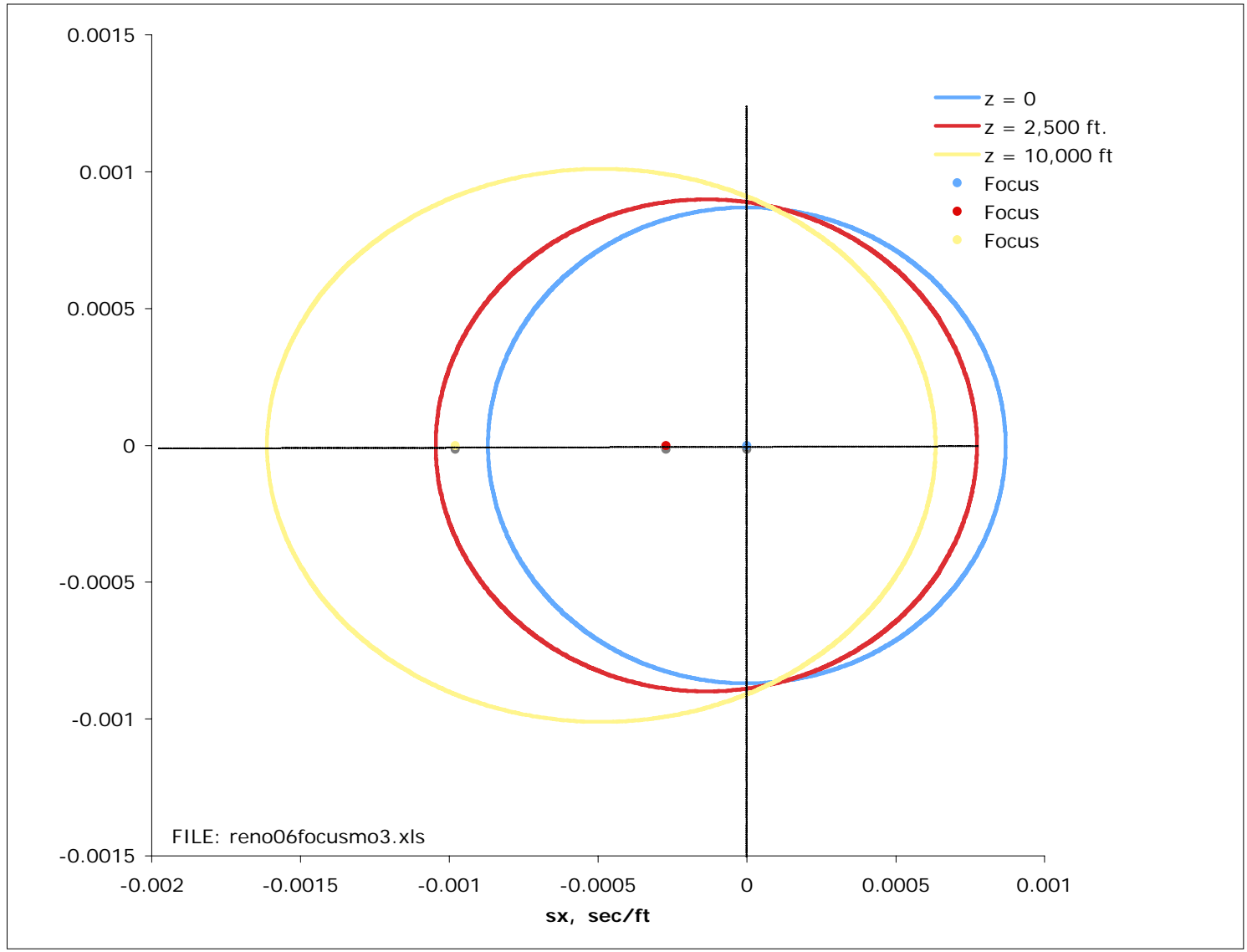

Figure 1. The motion of the second focus as $\mathrm{z}$ increases in an atmosphere within which the Mach number increases with altitude. The first focus is at $\left(\mathrm{s}_{\mathrm{x}}, \mathrm{s}_{\mathrm{y}}\right)=(0,0)$. $\stackrel{\mathrm{U}}{\mathrm{U}}=(0.024 \mathrm{z} / \mathrm{sec}) \hat{\mathrm{x}}, \mathrm{z}$ in feet. $\mathrm{c}=1100 \mathrm{ft} / \mathrm{sec}$.

\section{The Atmosphere as a Ray Filter}

For any value of $z$, that is for any altitude, equation (10) is the equation of an ellipse in the $\left(s_{x}, s_{y}\right)$ plane. This ellipse divides the $\left(s_{x}, s_{y}\right)$ plane into two regions. For points $\left(s_{x}, s_{y}\right)$ outside of the ellipse propagation is evanescent in the $\mathrm{z}$ direction. The interior of the ellipse consists of $\left(\mathrm{s}_{\mathrm{x}}, \mathrm{s}_{\mathrm{y}}\right)$ pairs that define rays that penetrate the plane. The boundary separating these regions, the ellipse itself, consists of $\left(\mathrm{s}_{\mathrm{x}}, \mathrm{s}_{\mathrm{y}}\right)$ pairs that define rays that turn at this value of $\mathrm{z}$. Thus, one way to look at the atmosphere, at least as to how it affects an acoustic disturbance propagating through it, is as a set of these filters, one for each plane $\mathrm{z}=$ constant. Each filter either removes certain rays from the field at the observer, or turns these rays back towards the observation plane.

If all of the ETPs for a given atmosphere were drawn in the same $\left(\mathrm{s}_{\mathrm{x}}, \mathrm{s}_{\mathrm{y}}\right)$ plane, the plane would be divided into four regions, see Figure 2. For $\left(\mathrm{s}_{\mathrm{x}}, \mathrm{s}_{\mathrm{y}}\right)$ in region 1 , propagation in $\mathrm{z}$ is evanescent. These waves either grow or decay as they propagate from the source to the receiver. Thus, in the first case, these waves must be removed from the field by a judicious application of an initial condition, and they may be neglected in the second. 
Hence, these values of $\left(\mathrm{s}_{\mathrm{x}}, \mathrm{s}_{\mathrm{y}}\right)$ need not be considered. Any ray specified by $\left(\mathrm{s}_{\mathrm{x}}, \mathrm{s}_{\mathrm{y}}\right)$ in regions 2 and 3 will go through at least one turning point. These rays will be considered in more detail later. Finally, for any $\left(\mathrm{s}_{\mathrm{x}}, \mathrm{s}_{\mathrm{y}}\right)$ in region 4 , the specified ray will connect the source to some point on the ground without going through a turning point. Clearly, a robust ray tracing program would easily be provided if consideration were restricted to rays specified by $\left(\mathrm{s}_{\mathrm{x}}, \mathrm{s}_{\mathrm{y}}\right)$ in region 4 . However, in the following, we will take a closer look at regions 2 and 3 to show how mapping out these regions allows consideration of all relevant rays while easily determining rays that cannot contribute to the field at the observer plane.

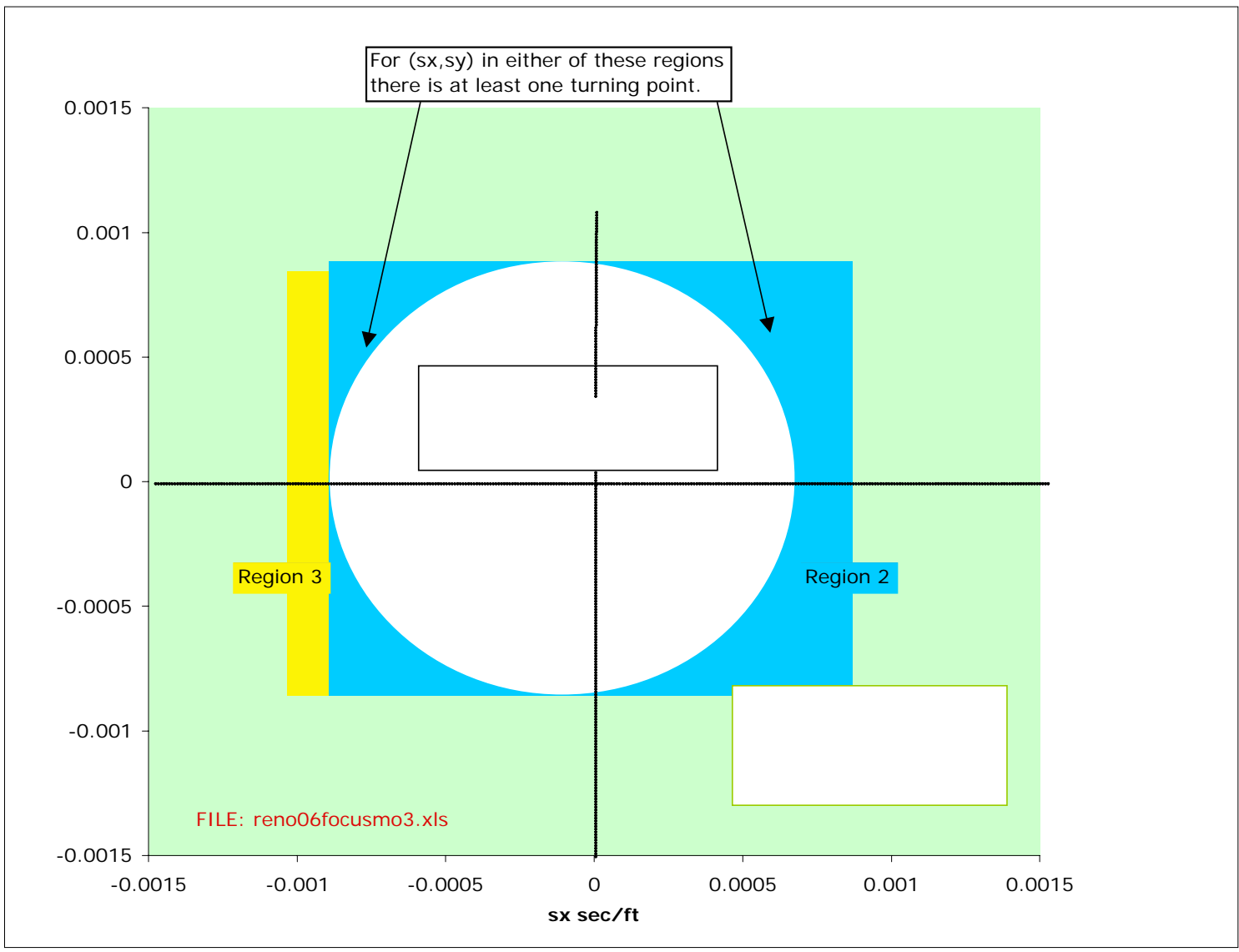

Figure 2. The set of all ellipses for a given atmosphere divides the $\left(\mathrm{s}_{\mathrm{x}}, \mathrm{s}_{\mathrm{y}}\right)$ plane into four regions.

\section{The Initial Refinement, Hacking Away at the Boundary}

If, instead of plotting all of the ellipses for a given atmosphere, a minimalist approach is taken, and only three ellipses, one each for the observer plane, the source plane, and the plane representing the top of the atmosphere, are drawn, a rather skeletal representation of Figure 2 is produced. This representation is presented in Figure 3. The three ellipses considered here represent the boundaries of significant regions. Since rays associated 
with $\left(\mathrm{s}_{\mathrm{x}}, \mathrm{s}_{\mathrm{y}}\right)$ in Region 2 of Figure 2 carry evanescent waves, points $\left(\mathrm{s}_{\mathrm{x}}, \mathrm{s}_{\mathrm{y}}\right)$ to the right of the ellipse for the source plane may be ignored. This suggests use of the right half of the source plane ellipse as part of the boundary of the region of interest. Further, points $\left(s_{x}, s_{y}\right)$ which fall outside the circle for the ground plane provide rays which cannot reach the ground. This suggests using the left half of the circle for the ground plane as the remainder of the boundary of the region of interest. Taking, then, the intersection of the regions $\mathrm{s}_{\mathrm{x}}^{2}+\mathrm{s}_{\mathrm{y}}^{2} \leq \frac{1}{\mathrm{c}_{0}^{2}}$ and $\mathrm{s}_{\mathrm{x}}^{2}+\mathrm{s}_{\mathrm{y}}^{2} \leq \frac{\left[1-\left(\mathrm{U}_{\mathrm{s}} \mathrm{s}_{\mathrm{x}}+\mathrm{V}_{\mathrm{s}} \mathrm{s}_{\mathrm{y}}\right)\right]^{2}}{\mathrm{c}_{\mathrm{s}}^{2}}$ yields a region in the $\left(\mathrm{s}_{\mathrm{x}}, \mathrm{s}_{\mathrm{y}}\right)$ plane that more closely approximates the region containing all $\left(\mathrm{s}_{\mathrm{x}}, \mathrm{s}_{\mathrm{y}}\right)$ pairs that determine rays that can contribute to the field at the observer plane. This region is shown in Figure 4 , which represents a first refinement of the region suggested earlier.

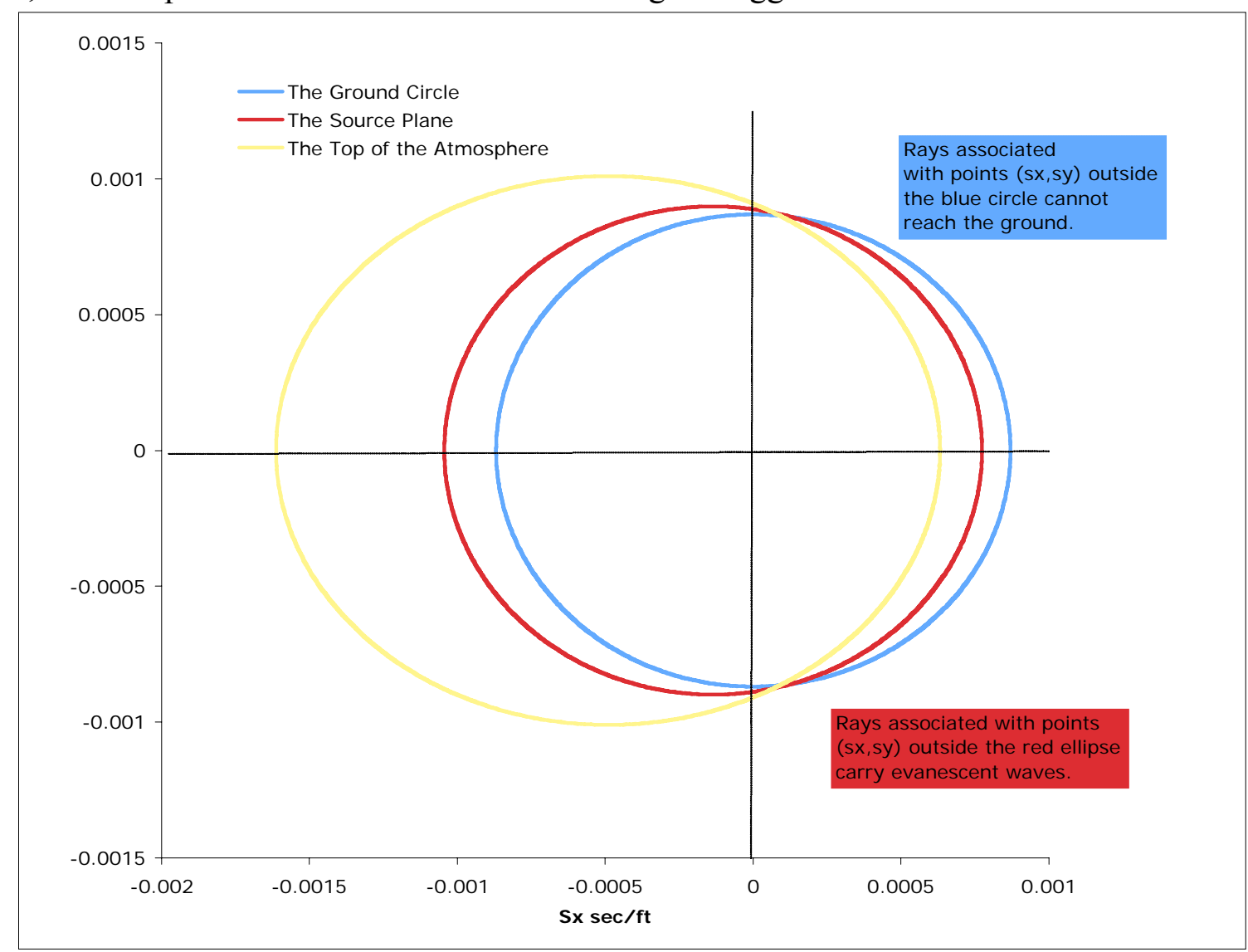

Figure 3. A skeletal representation of the elliptical acoustic filters for an atmosphere. 


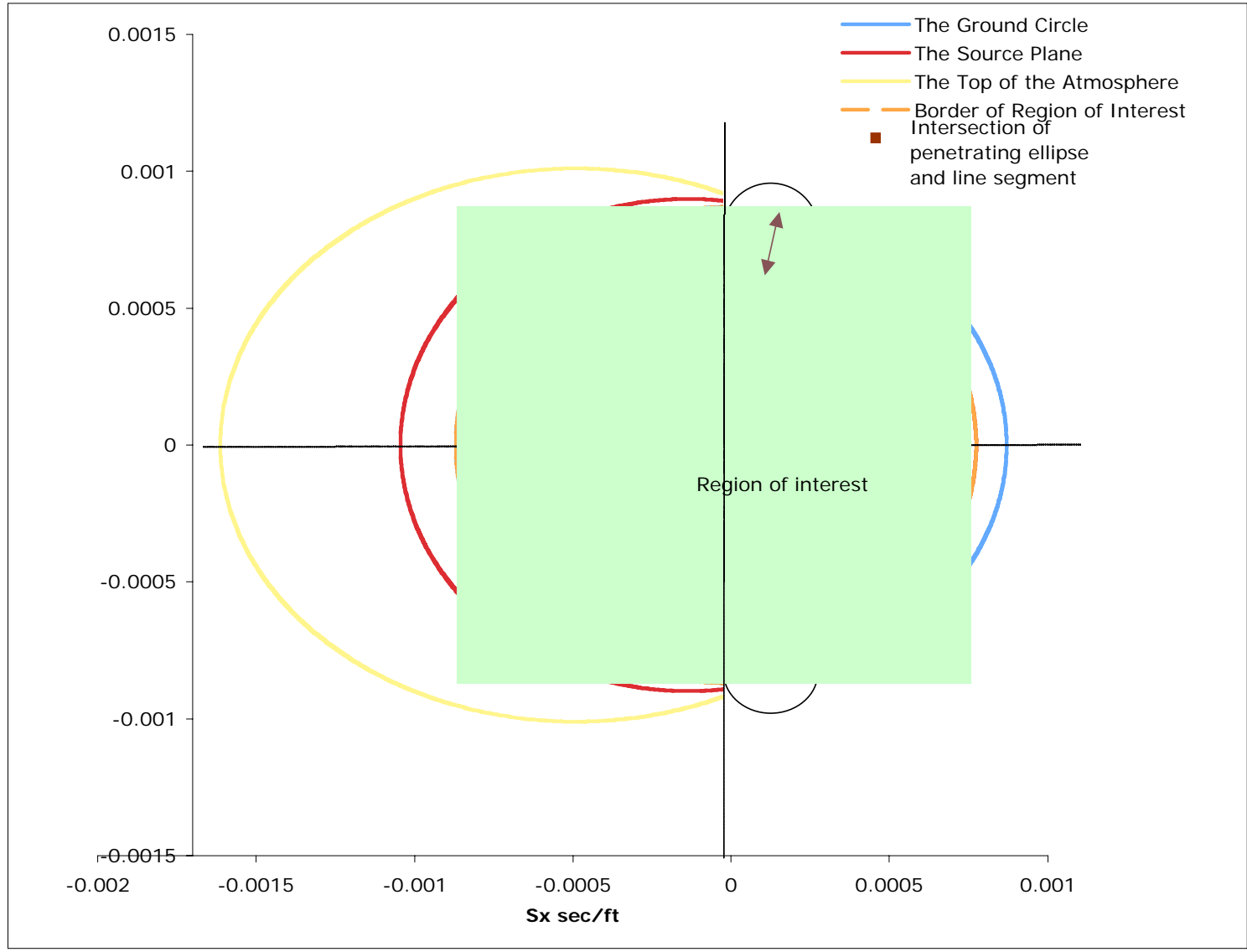

Figure 4. The region $\left(\mathrm{s}_{\mathrm{x}}, \mathrm{s}_{\mathrm{y}}\right)$ for rays that will certainly reach the ground, and two regions requiring further analysis.

Except for points $\left(\mathrm{s}_{\mathrm{x}}, \mathrm{s}_{\mathrm{y}}\right)$ located within the small areas of concern indicated in Figure 4, all points $\left(\mathrm{s}_{\mathrm{x}}, \mathrm{s}_{\mathrm{y}}\right)$ in this region provide rays that will reach the ground. Further, all points $\left(\mathrm{s}_{\mathrm{x}}, \mathrm{s}_{\mathrm{y}}\right)$ outside of this region either fall outside of the circle $\mathrm{s}_{\mathrm{x}}^{2}+\mathrm{s}_{\mathrm{y}}^{2}=\mathrm{c}_{0}^{-2}$, and therefore determine rays that cannot reach the ground, or fall outside of the ellipse $\mathrm{s}_{\mathrm{x}}^{2}+\mathrm{s}_{\mathrm{y}}^{2}=\left[1-\left(\mathrm{U}_{\mathrm{s}} \mathrm{s}_{\mathrm{x}}+\mathrm{Vs}_{\mathrm{y}}\right)\right]^{2} / \mathrm{c}_{\mathrm{s}}^{2}$, and, therefore, determine rays which carry evanescent waves at the source. Generally, these may safely be ignored. Hence, consideration may be limited to points $\left(\mathrm{s}_{\mathrm{x}}, \mathrm{s}_{\mathrm{y}}\right)$ in the indicated region.

\section{The Final Refinement}

Now consider the regions indicated as "Areas of concern" in Figure 4. Within these regions, it is conceivable that an ellipse associated with an altitude between that of the source and the ground passes through our region of interest. The points $\left(s_{x}, s_{y}\right)$ falling on this ellipse would then be associated with rays passing through a turning point. These points $\left(\mathrm{s}_{\mathrm{x}}, \mathrm{s}_{\mathrm{y}}\right)$, then, are associated either with ducted rays or with rays that pass through a turning point and do not reach the ground. Consider the line segment between the origin $\left(s_{x}, s_{y}\right)=(0,0)$ and an arbitrary point on the border of the region of interest indicated in Figure 4. Let this line segment be at an angle $\alpha$ to the positive $s_{x}$ axis. Then, the intersection of any ellipse entering the region of interest with this line segment will be a 
point. As $\mathrm{z}$ varies, $0 \leq \mathrm{z} \leq \mathrm{z}_{\mathrm{m}}$, where $\mathrm{z}_{\mathrm{m}}$ is the altitude of the top of the atmosphere, this point will move along the line segment, as indicated in Figure 4. The vector from the origin to this moving point will be denoted by $\stackrel{v}{s}_{t}$. Thus, the equation

$$
\stackrel{\mathbf{s}_{\mathrm{t}}}{\mathbf{s}_{\mathrm{t}}}=\left|\stackrel{\mathbf{v}}{\mathbf{s}_{\mathrm{t}}}\right|[\hat{\mathrm{x}} \operatorname{Cos}(\alpha)+\hat{\mathrm{y}} \operatorname{Sin}(\alpha)]
$$

must hold. Further, since this point lies on an ETP, $\mathrm{s}_{\mathrm{z}}=0$, and it must also be true that

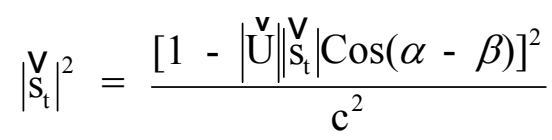

There are two values of $\left|\mathrm{S}_{t}\right|$ that satisfy this equation, but for subsonic flow the only physically valid root is

$$
\mid \begin{aligned}
& \mathbf{v}_{\mathrm{t}} \\
& \mathbf{s}
\end{aligned}=\frac{1}{[\mathrm{c}+|\mathrm{U}| \operatorname{Cos}(\alpha-\beta)]}
$$

Differentiating equation (13) with-respect-to $\mathrm{z}$ while holding $\alpha$ constant, and setting $\frac{\mathrm{d}\left|\mathbf{s}_{\mathrm{t}}\right|}{\mathrm{dz}}=0$, yields

$$
\frac{\mathrm{d} \dot{U}}{\mathrm{dz}} \cdot \frac{\stackrel{\mathrm{s}}{\mathrm{t}}_{\mathrm{v}}^{\mathrm{v}}}{\left|\mathrm{S}_{\mathrm{t}}\right|}+\frac{\mathrm{dc}}{\mathrm{dz}}=0
$$

Elimination of $\mathrm{z}$ between equation (13) and equation (14) yields an equation for the minimum value of $\left|\mathbf{s}_{t}\right|$ as a function of $\alpha$. The curve so generated, shown in Figure 5, is the envelope of the ellipses that sweep through the circle $\mathrm{s}_{\mathrm{x}}^{2}+\mathrm{s}_{\mathrm{y}}^{2}=\mathrm{c}_{0}^{-2}$. There is, obviously, more to the envelope curve than is shown here. However, all that is really of interest are the segments of the envelope curve between where it is tangent to the circle $\mathrm{s}_{\mathrm{x}}^{2}+\mathrm{s}_{\mathrm{y}}^{2}=\mathrm{c}_{0}^{-2}$ and where it is tangent to the ellipse $\mathrm{s}_{\mathrm{x}}^{2}+\mathrm{s}_{\mathrm{y}}^{2}=\left[1-\left(\mathrm{U}_{\mathrm{z}_{\mathrm{m}}} \mathrm{s}_{\mathrm{x}}+\mathrm{V}_{\mathrm{z}_{\mathrm{m}}} \mathrm{s}_{\mathrm{y}}\right)\right]^{2} / \mathrm{c}_{\mathrm{z}_{\mathrm{m}}}^{2}$. Here, the subscript $\mathrm{z}_{\mathrm{m}}$ indicates evaluation of the indicated quantity at the top of the atmosphere. There are two of these segments, one each for the two areas of concern indicated in Figure 4. For the case considered here, however, these are mirror images of each other. Therefore, only the upper segment is shown in Figure 5. 


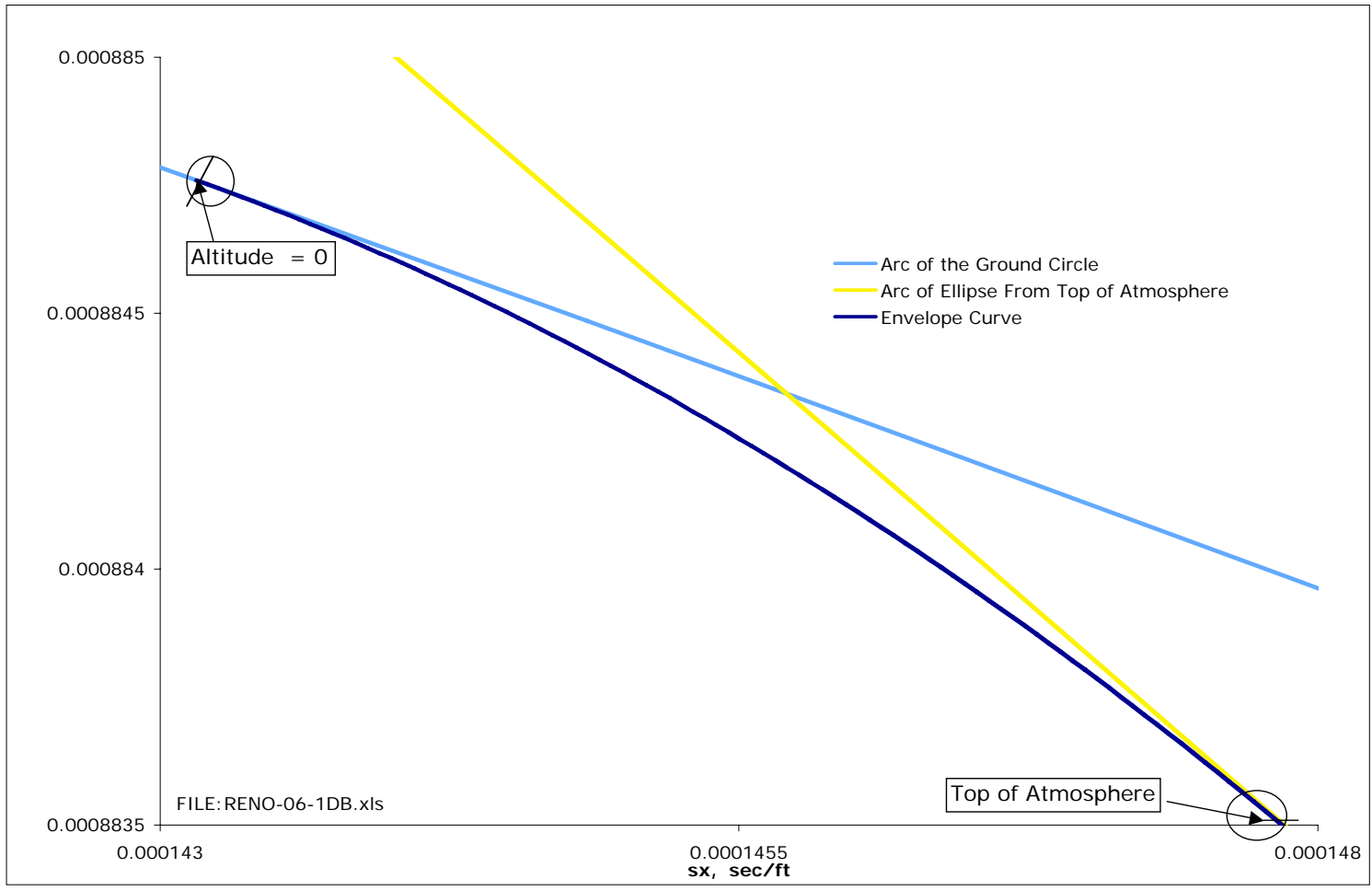

Figure 5. The envelope curve obtained by elimination of $z$ between equations (13) and (14) connects the circle $s_{x}^{2}+s_{y}^{2}=c_{0}^{-2}$ with the ellipse $\mathrm{s}_{\mathrm{x}}^{2}+\mathrm{s}_{\mathrm{y}}^{2}=\left[1-\left(\mathrm{U}_{\mathrm{z}_{\mathrm{m}}} \mathrm{s}_{\mathrm{x}}+\mathrm{V}_{\mathrm{z}_{\mathrm{m}}} \mathrm{s}_{\mathrm{y}}\right)\right]^{2} \mathrm{c}_{\mathrm{z}_{\mathrm{m}}}^{-2}$. Note that each point on this curve is associated with a value of $z, 0 \leq z \leq z_{m}$, and that its end points are associated with $z=0$, the ground, and $\mathrm{z}=\mathrm{z}_{\mathrm{m}}$, the top of the atmosphere, as indicated in the figure.

The two points at which the envelope curve is tangent to any ellipse divide that ellipse into two segments. Along the envelope curve, hence at the point where the envelope curve is tangent to the ellipse, equation (14) is satisfied. Therefore, along one of the two segments of the ellipse it must be true that

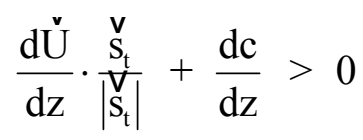

while along the other the inequality

$$
\frac{d \dot{U}}{d z} \cdot \frac{\stackrel{s}{s}_{t}}{\left|S_{t}\right|}+\frac{d c}{d z}<0
$$

must hold. Consider a wave approaching the turning point from below, as shown on the left-hand-side of Figure 6 . In this case, $s_{z}^{2}$, while zero at $z=z_{t}$, must be greater than zero in $\mathrm{z}<\mathrm{z}_{\mathrm{t}}$, at least near $\mathrm{z}=\mathrm{z}_{\mathrm{t}}$. Generally, in this case, $\mathrm{s}_{\mathrm{z}}^{2}<0$ in $\mathrm{z}>\mathrm{z}_{\mathrm{t}}$. Hence, we must have $\mathrm{d}\left(\mathrm{s}_{\mathrm{z}}^{2}\right) / \mathrm{dz}<0$ at $\mathrm{z}=\mathrm{z}_{\mathrm{t}}$. Differentiating Equation (13) with-respect-to $\mathrm{z}$, and requiring 
$\mathrm{d}\left(\mathrm{s}_{\mathrm{z}}^{2}\right) / \mathrm{dz}<0$, yields inequality (15). Similarly, if we consider a wave approaching the turning point from above, as shown on the right-hand-side of Figure 6, we must have $\mathrm{d}\left(\mathrm{s}_{\mathrm{z}}^{2}\right) / \mathrm{dz}>0$, and it may be shown that inequality (16) must hold. Therefore, the points where the envelope curve is tangent to the ellipse divide that ellipse into two segments. Along one of these segments the corresponding rays approach the turning point from below, with evanescent propagation above the turning point, whereas along the other segment the corresponding rays approach the turning point from above, with evanescent propagation below the turning point. Figures 7 and 8 summarize these results.

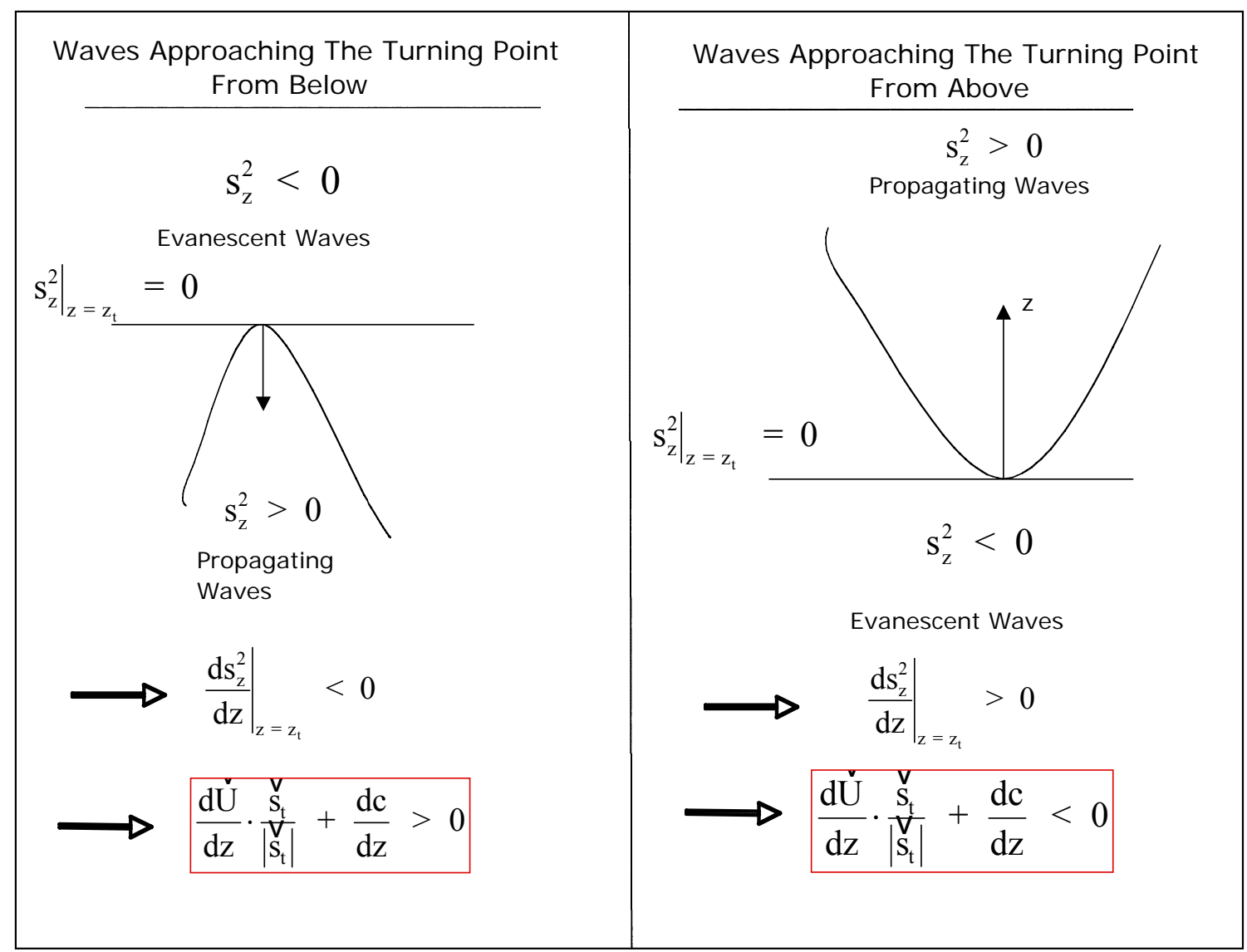

Figure 6. The inequalities (15) and (16) and their implications for the direction of a ray at a turning point. 


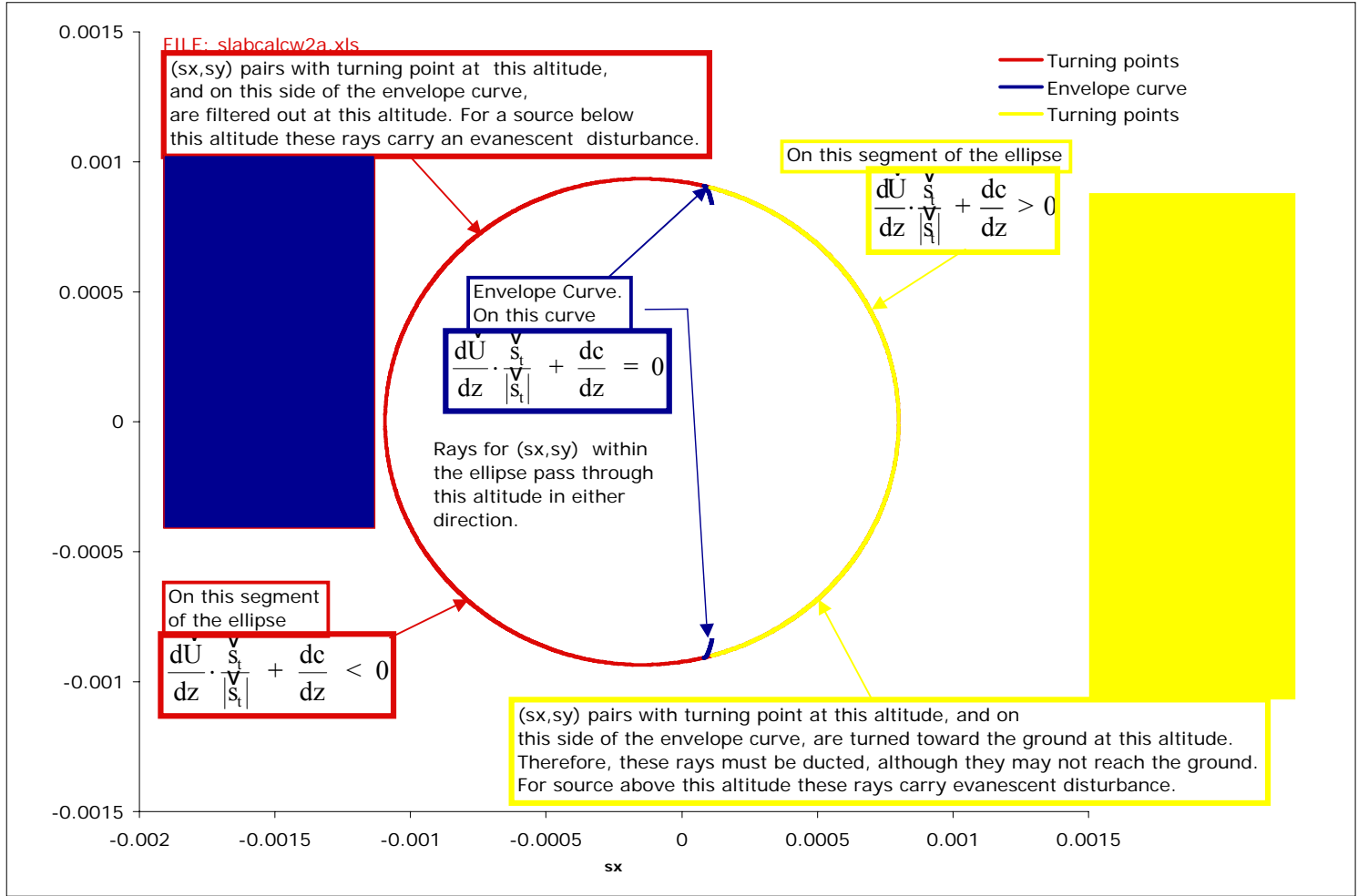

Figure 7. How the envelope curve divides any given ellipse of turning points, and the implied direction of the ray.

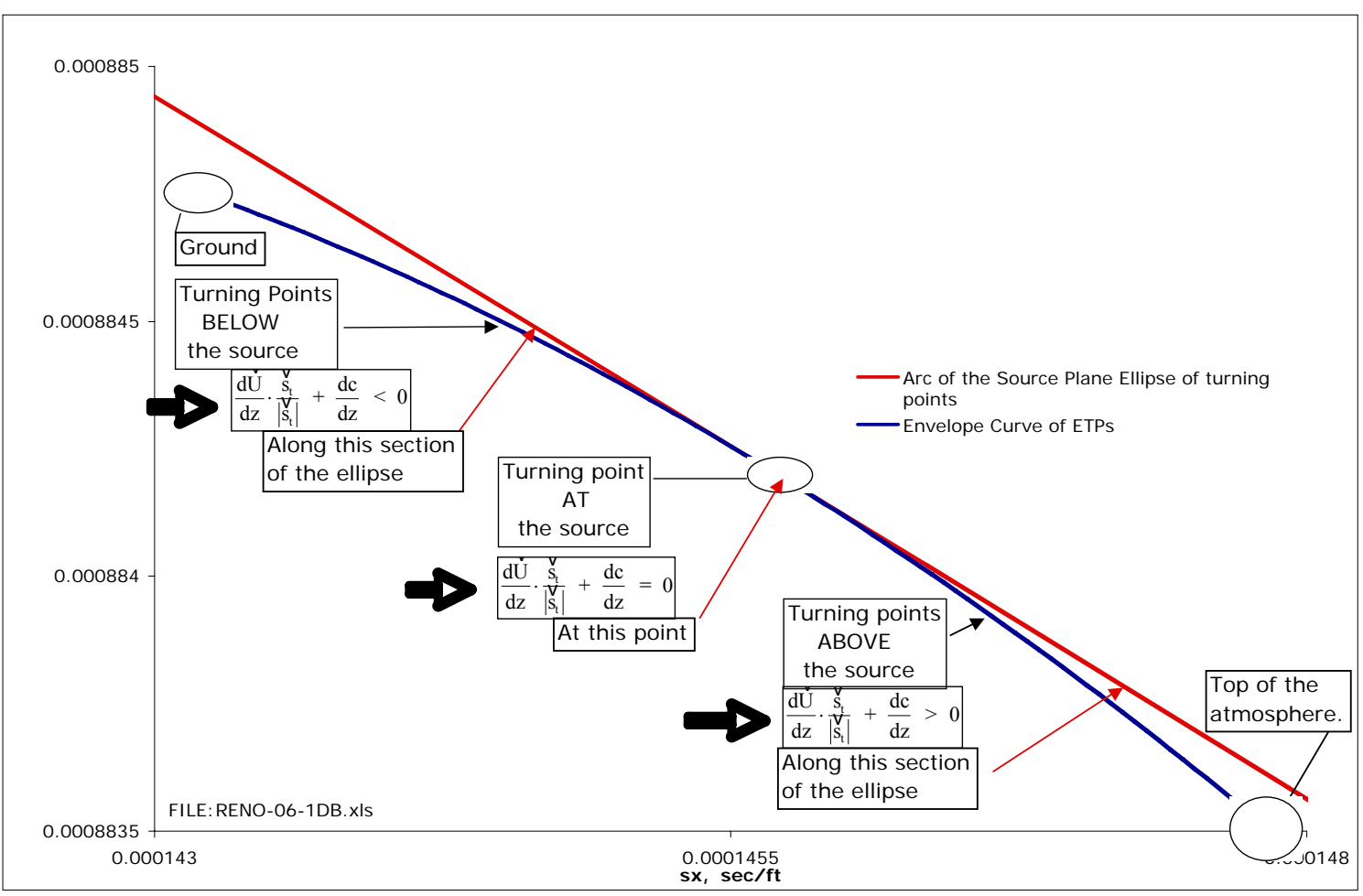

Figure 8. A closer look at the neighborhood of the point where the envelope curve is tangent to the source plane ellipse of turning points. 


\section{IIX. The Ray Behavior For Each Region}

It is now possible to give a rather complete description of the characteristics of a ray determined by any point in the $\left(\mathrm{s}_{\mathrm{x}}, \mathrm{s}_{\mathrm{y}}\right)$ plane. It is suggested that the reader refer to Figures 9a-c while reading the following description. Figure 9a provides a close-up view of the upper "Region of concern" shown in Figure 4, while Figure 9c provides a close-up view of the lower "Region of concern" of that figure. Figure $9 \mathrm{~b}$ provides a birds-eye view of the $\left(s_{x}, s_{y}\right)$ plane. Note that regions referred to by number are regions seen, in some sense, when a rather gross view of the $\left(\mathrm{s}_{\mathrm{x}}, \mathrm{s}_{\mathrm{y}}\right)$ plane is taken. Regions referred to by letter are regions that would be seen only upon a rather close look at the $\left(s_{x}, s_{y}\right)$ plane.

Referring to Figure 9b, points $\left(s_{x}, s_{y}\right)$ in Region 1 are outside of all of the ellipses for points within the modeled atmosphere. Thus, for these points $\left(s_{x}, s_{y}\right), s_{z}$ is imaginary for all $\mathrm{z}$. Propagation is, therefore, evanescent in $\mathrm{z}$. These values of $\left(\mathrm{s}_{\mathrm{x}}, \mathrm{s}_{\mathrm{y}}\right)$ may be neglected.

For points $\left(\mathrm{s}_{\mathrm{x}}, \mathrm{s}_{\mathrm{y}}\right)$ in Region $2, \mathrm{~s}_{\mathrm{z}}$ is imaginary at all altitudes between the source and the observer. These values of $\left(\mathrm{s}_{\mathrm{x}}, \mathrm{s}_{\mathrm{y}}\right)$ may be neglected.

For points $\left(\mathrm{s}_{\mathrm{x}}, \mathrm{s}_{\mathrm{y}}\right)$ in Region $3, \mathrm{~s}_{\mathrm{z}}$ is real at the source, but imaginary at the observer plane. Therefore, these rays must pass through a turning point between the source and the observer, and do not reach the ground. These values of $\left(\mathrm{s}_{\mathrm{x}}, \mathrm{s}_{\mathrm{y}}\right)$ may be neglected.

For points $\left(\mathrm{s}_{\mathrm{x}}, \mathrm{s}_{\mathrm{y}}\right)$ in Region $4, \mathrm{~s}_{\mathrm{z}}$ is real both at the source and at the observer plane. Further, except for a small subset of points $\left(\mathrm{s}_{\mathrm{x}}, \mathrm{s}_{\mathrm{y}}\right)$ within this region, and considered later, $s_{z}$ is real at all altitudes between the source and the observer. Thus, except for a small subset of $\left(\mathrm{s}_{\mathrm{x}}, \mathrm{s}_{\mathrm{y}}\right)$ in this region, these rays carry energy from the source to the observer plane.

For points $\left(\mathrm{s}_{\mathrm{x}}, \mathrm{s}_{\mathrm{y}}\right)$ in Region $5, \mathrm{~s}_{\mathrm{z}}$ is real at both the source plane and the observer plane, and at all points between the source and the observer. Once again, these rays carry energy from the source to the observer plane. However, for these points $\left(\mathrm{s}_{\mathrm{x}}, \mathrm{s}_{\mathrm{y}}\right)$, a turning point occurs somewhere above the source altitude. Therefore, these rays are ducted, and multipathing occurs.

For points $\left(\mathrm{s}_{\mathrm{x}}, \mathrm{s}_{\mathrm{y}}\right)$ in Region $6, \mathrm{~s}_{\mathrm{z}}$, although real at the observer plane, is imaginary at the source. These points $\left(\mathrm{s}_{\mathrm{x}}, \mathrm{s}_{\mathrm{y}}\right)$ need not be considered.

We now take a closer look at the regions of concern of Figure 4. The reader should consult Figures 9a and 9c during this discussion.

Region A is inside both the ground circle and the ellipse for the source plane. Further, no turning points have entered this region, as it lies to the left of the envelope curve. Region 
$A$ is, in fact, Region 4 discussed above. Each ray associated with a point $\left(s_{x}, s_{y}\right)$ within this region carries energy from the source to the plane $\mathrm{z}=0$.

Region B is outside of the ground circle, but inside the source ellipse. Therefore, $\mathrm{s}_{\mathrm{z}}$ is real at the source, but imaginary at the ground. Hence, there is at least one turning point between the source and the ground plane for points $\left(\mathrm{s}_{\mathrm{x}}, \mathrm{s}_{\mathrm{y}}\right)$ within this region. The ray is turned away from the observation plane at this turning point. Therefore, these pairs $\left(\mathrm{s}_{\mathrm{x}}, \mathrm{s}_{\mathrm{y}}\right)$ need not be considered.

Regions $\mathrm{C}$ and $\mathrm{E}$ are both within both the source ellipse and the circle for the ground plane. Thus, $s_{z}$ is real at both the source and the receiver. However, the behavior of the ray is different for a ray associated with a point in region $\mathrm{C}$ than it is for a ray associated with a point in Region E. Referring to Figure 8 it may be seen that the turning point for a ray from Region $\mathrm{C}$ is below the source, while the turning point for a ray from Region $\mathrm{E}$ is above the source. Thus, rays from Region $\mathrm{C}$ are turned away from the observation plane while those from Region $\mathrm{E}$ are turned toward the observation plane. Therefore, rays from Region $\mathrm{C}$ may be ignored while those from Region $\mathrm{E}$ are ducted between an altitude at or above the source plane and the ground and contribute to the disturbance field. Note that for rays associated with points in Region E there are two turning points above the source altitude. At the first, the ray is turned back toward the source, hence the ground. At the second, a ray is turned away from the ground.

Region D is within the ground circle, but outside the source plane ellipse. Therefore, for points $\left(s_{x}, s_{y}\right)$ within Region $D, s_{z}$ is imaginary at the source, although real at the observer. Again, these pairs $\left(s_{x}, s_{y}\right)$ may be neglected.

Region $\mathrm{F}$ is within the ellipse for the source plane, and inside of the circle for the ground plane. Further, Region F is outside of the envelope curve. Note, however, that Region F is also outside of the ellipse for the top of the atmosphere. Rays associated with points within Region $\mathrm{F}$ go through a turning point at an altitude above the source plane. In contrast to points in Region $\mathrm{E}$, however, there is only one turning point above the source for rays associated with points in Region F. Region F is, in fact, Region 5. These rays are ducted and contribute to the disturbance field on the observer plane.

For points $\left(\mathrm{s}_{\mathrm{x}}, \mathrm{s}_{\mathrm{y}}\right)$ within Region $\mathrm{G}, \mathrm{s}_{\mathrm{z}}$ is real at the ground, but imaginary at the source. These rays may be ignored.

For points $\left(\mathrm{s}_{\mathrm{x}}, \mathrm{s}_{\mathrm{y}}\right)$ within Region $\mathrm{H}, \mathrm{s}_{\mathrm{z}}$ is imaginary for all points within the atmosphere. Thus, Region H is Region 1.

For Region $\mathrm{I}, \mathrm{s}_{\mathrm{Z}}$ is imaginary for all points between the source and the observer. This is Region 2.

In summary, only rays from Region E, in addition to rays from Regions 4 and 5 considered previously, will reach the ground and add to the disturbance field. Rays from all other regions should be ignored from the outset. 


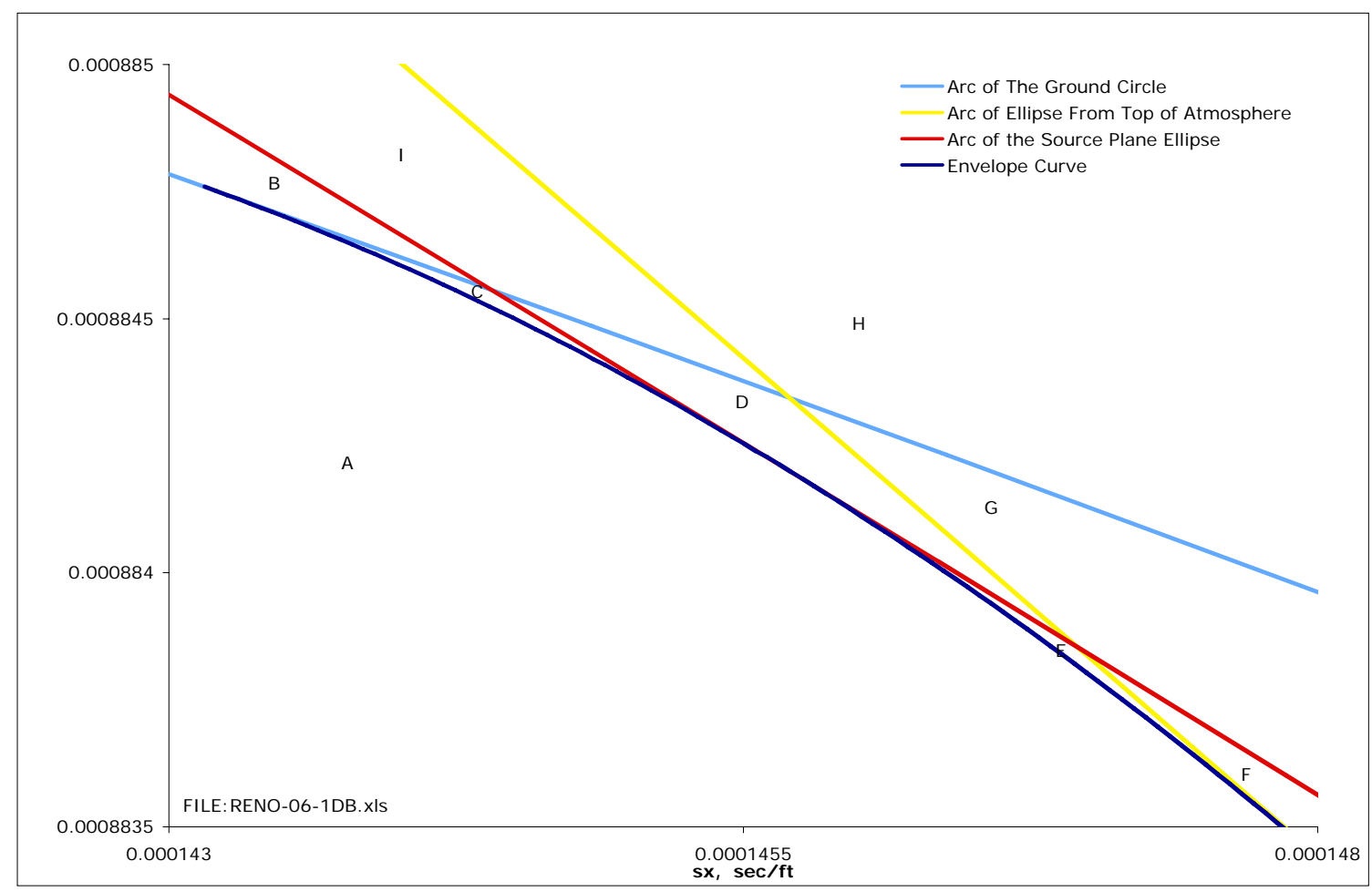

Figure 9a. Magnified view of the upper Area of concern from Figure 4 and the regions formed in that plane by the ground circle, source plane ellipse, the ellipse for the top of the atmosphere, and the envelope curve. 


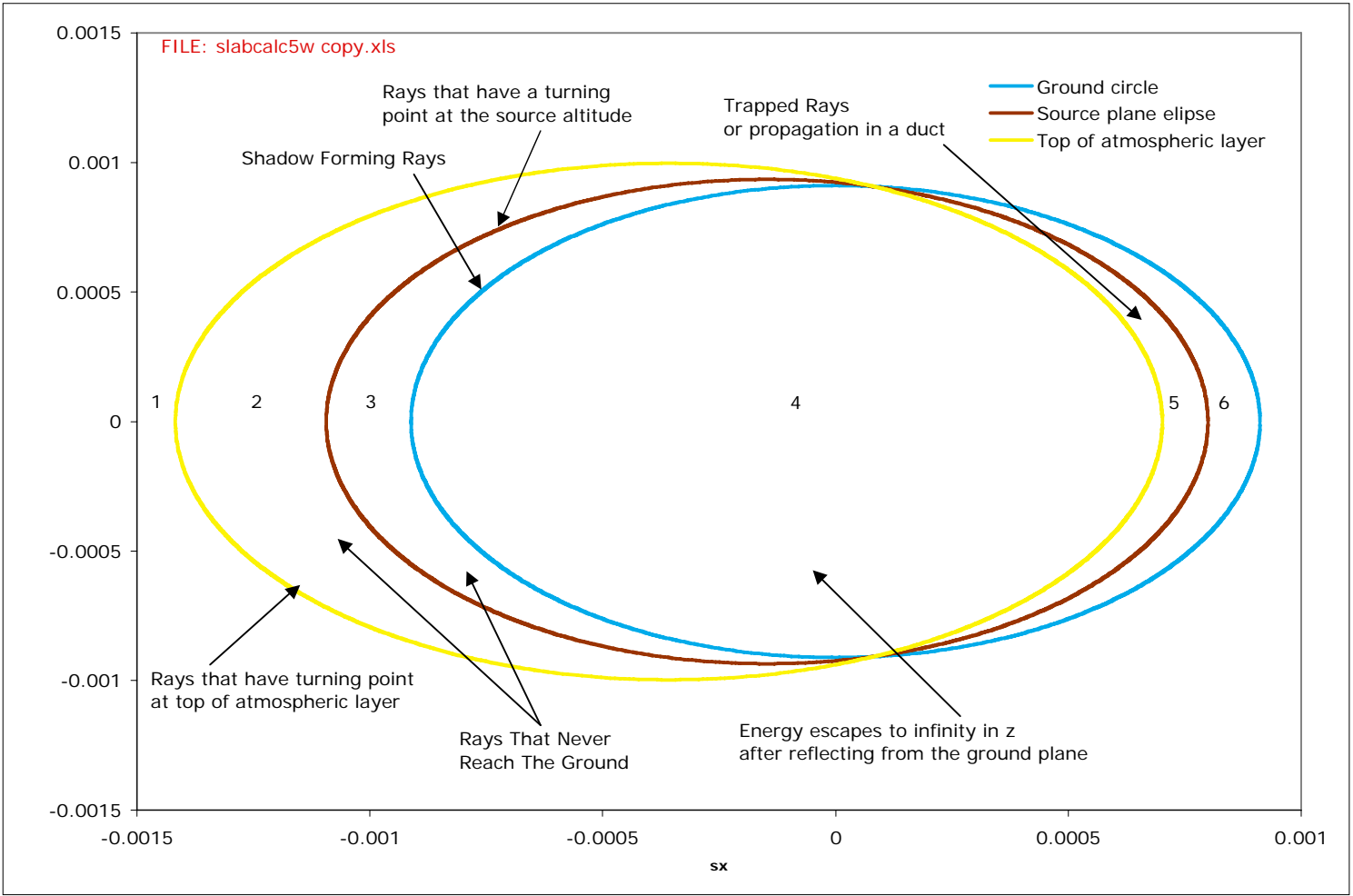

Figure $9 \mathrm{~b}$. An overview of the $\left(\mathrm{s}_{\mathrm{x}}, \mathrm{s}_{\mathrm{y}}\right)$ plane and the regions formed in that plane by the ground circle, source plane ellipse, and the ellipse for the top of the atmosphere. 
9-14 January 20006, Reno, Nevada

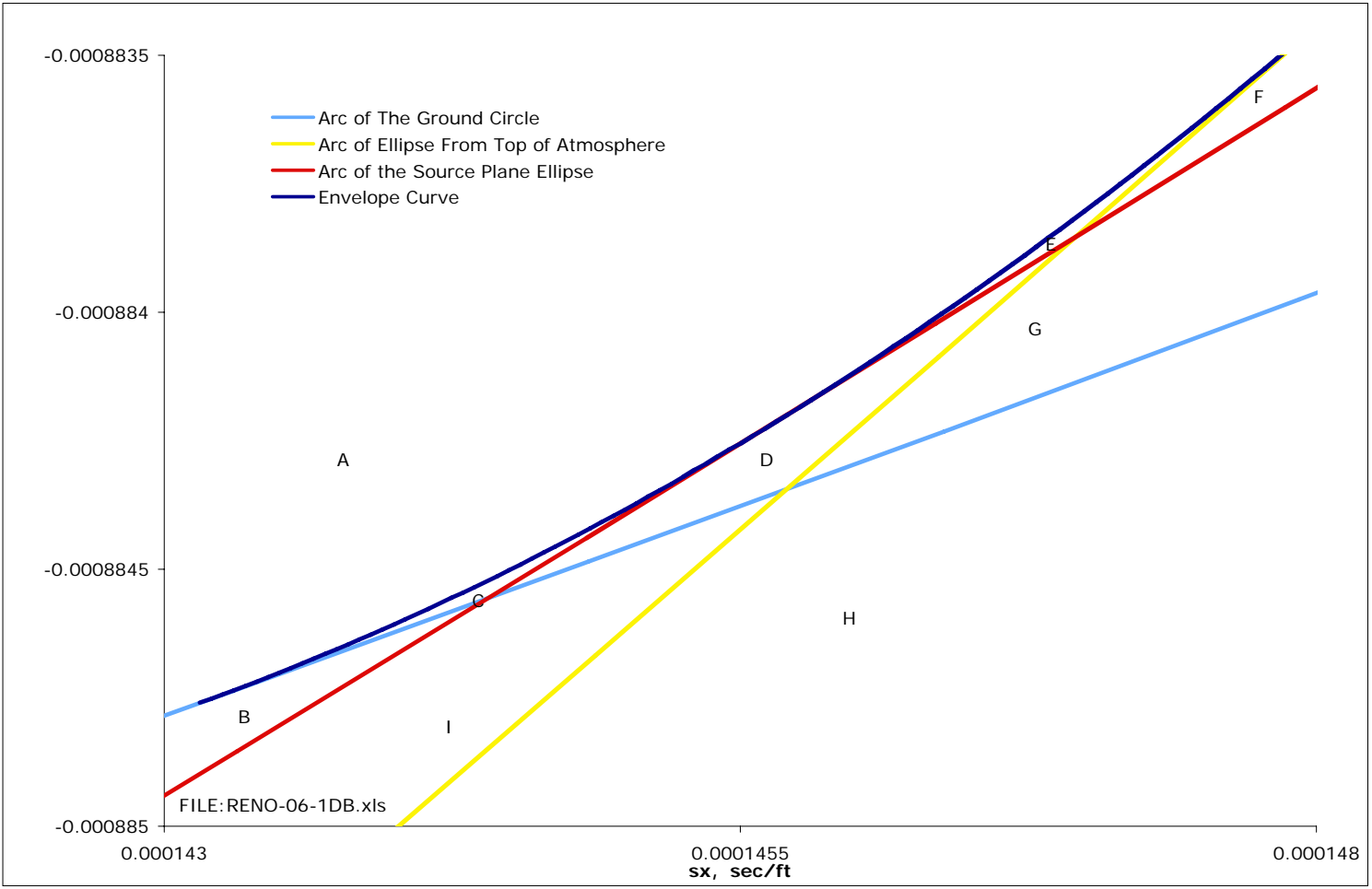

Figure 9c. Magnified view of the lower Area of concern from Figure 4 and the regions formed in that plane by the ground circle, source plane ellipse, the ellipse for the top of the atmosphere, and the envelope curve. 


\section{The Shadow Forming Rays}

In all that follows, it will be assumed that the observer is on the ground, which is located at $\mathrm{z}=0$, and that a no-slip condition is satisfied by the mean flow. Shadow forming rays are those for which $s_{z}=0$ at the ground. Thus, for shadow forming rays, equation (9), reproduced here for convenience, requires that

$$
\mathrm{s}_{\mathrm{x}}^{2}+\mathrm{s}_{\mathrm{y}}^{2}=\frac{1}{\mathrm{c}_{0}^{2}}
$$

where, as indicated previously, $\mathrm{c}_{0}$ is the sound speed at $\mathrm{z}=0$. Of course, only values of $\mathrm{s}_{\mathrm{x}}$ and $\mathrm{s}_{\mathrm{y}}$ that satisfy equation (9) are associated with rays that contribute to the shadow boundary. However, not all pairs $\left(\mathrm{s}_{\mathrm{x}}, \mathrm{s}_{\mathrm{y}}\right)$ satisfying equation (9) will contribute to the shadow boundary simply because some of these points may be associated with rays that pass through a turning point between the source and the ground plane and, therefore, never reach the ground. Further, although the atmosphere between the source and the observer can filter out rays that belong to this set, and thereby remove points from the shadow boundary, this intervening atmosphere can never cause rays that are not members of this set to contribute to the shadow boundary. Therefore, the shadow boundary is easily determined by tracing rays associated with points $\left(\mathrm{s}_{\mathrm{x}}, \mathrm{s}_{\mathrm{y}}\right)$ belonging to this set. Further, if certain members of this set are associated with rays that cannot reach the ground, these rays cannot contribute to the shadow boundary and there is no need to trace them.

Consider a case in which the temperature decreases linearly with altitude. Then, a circular shadow boundary will form as shown in Figure 10. Since it is known that this shadow boundary is circular, it is easily determined by tracing a single ray. However, it should be remembered that each point $(\mathrm{x}, \mathrm{y})$ on the shadow boundary is associated with a single point $\left(\mathrm{s}_{\mathrm{x}}, \mathrm{s}_{\mathrm{y}}\right)$ in the $\left(\mathrm{s}_{\mathrm{x}}, \mathrm{s}_{\mathrm{y}}\right)$ plane.

If a unidirectional mean flow with a speed increasing linearly with altitude is imposed, some of the shadow forming rays pass through a turning point before reaching the ground and are, therefore, removed form the set of shadow forming rays. The rays removed from the shadow boundary are on the segment of the source plane ellipse along which $\frac{\mathrm{dU}}{\mathrm{dz}} \cdot \frac{\stackrel{\mathrm{s}}{\mathrm{t}}^{\mathrm{v}}}{\left|\mathrm{S}_{\mathrm{t}}\right|}+\frac{\mathrm{dc}}{\mathrm{dz}}>0$, as discussed earlier. Eliminating these values of $\left(\mathrm{s}_{\mathrm{x}}, \mathrm{s}_{\mathrm{y}}\right)$ from the set of rays traced to obtain the shadow boundary, yields the arc shown in Figure 11. The resulting shadow boundary is shown in Figure 12. Naive geometric acoustic theory cannot predict the disturbance field at observer locations within the shadow region. Therefore, determination of the shadow boundary allows quick determination of observers for which the theory cannot be applied and prevents attempts to trace rays to observers for which no eigenray exists, thus saving computer time. 


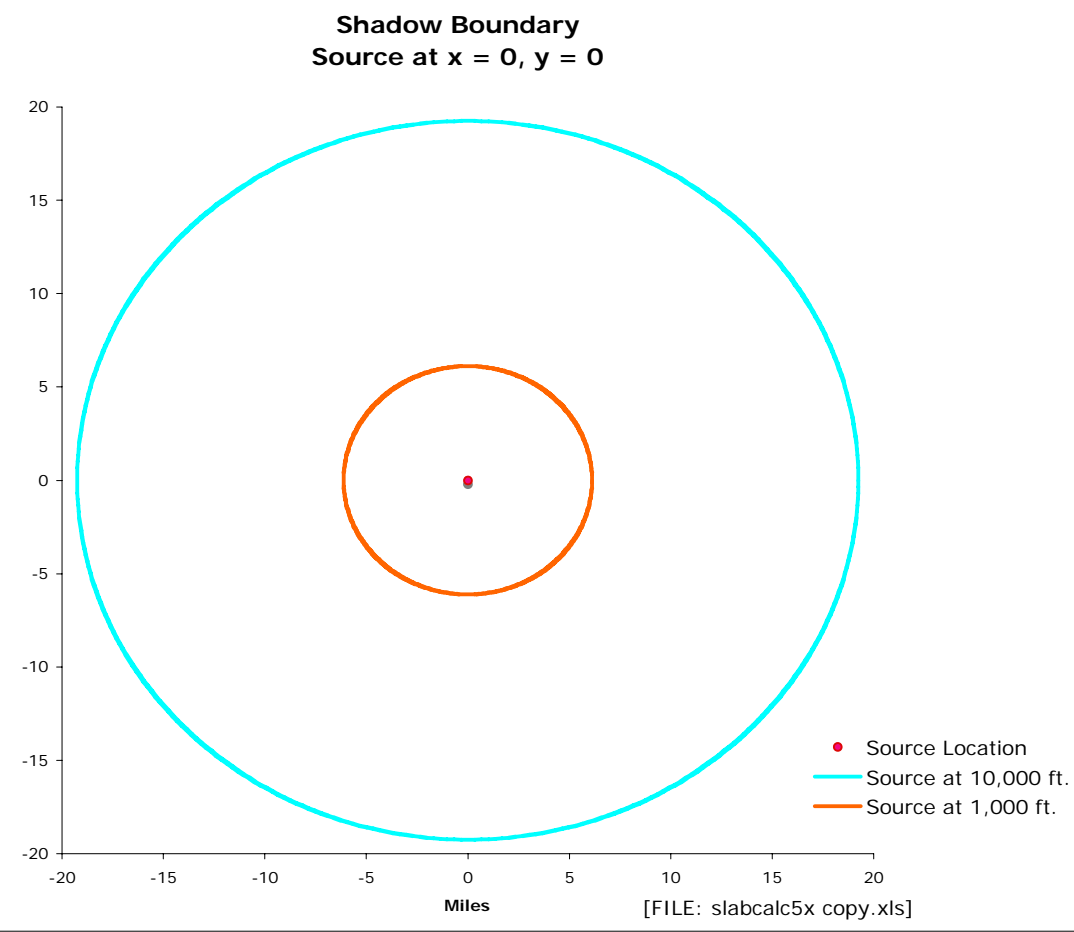

Figure 10. The circular shadow boundary for a point source in a medium within which the temperature decreases linearly with altitude.

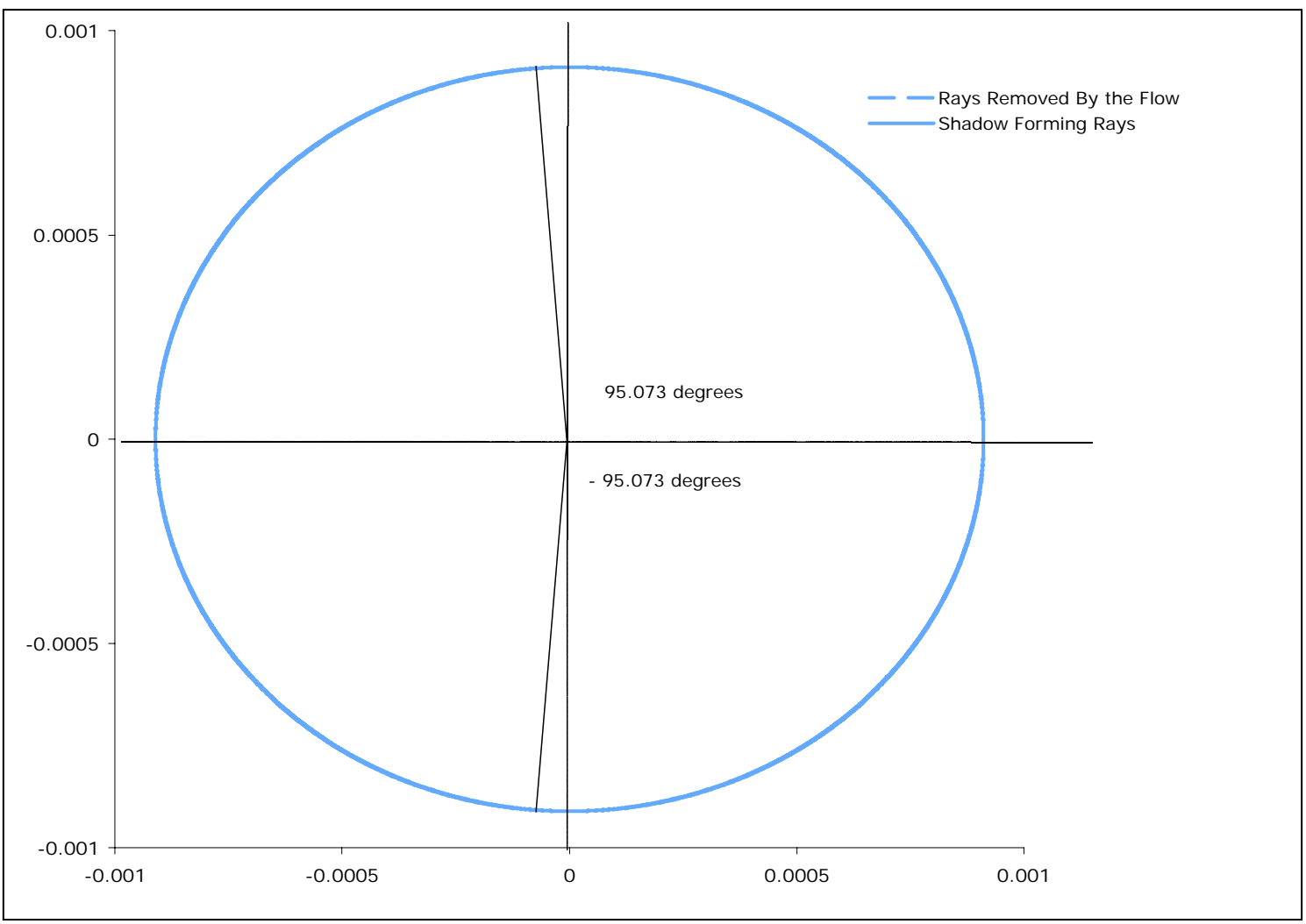

Figure 11. The arc of pairs $\left(\mathrm{s}_{\mathrm{x}}, \mathrm{s}_{\mathrm{y}}\right)$ that yields the shadow boundary. 


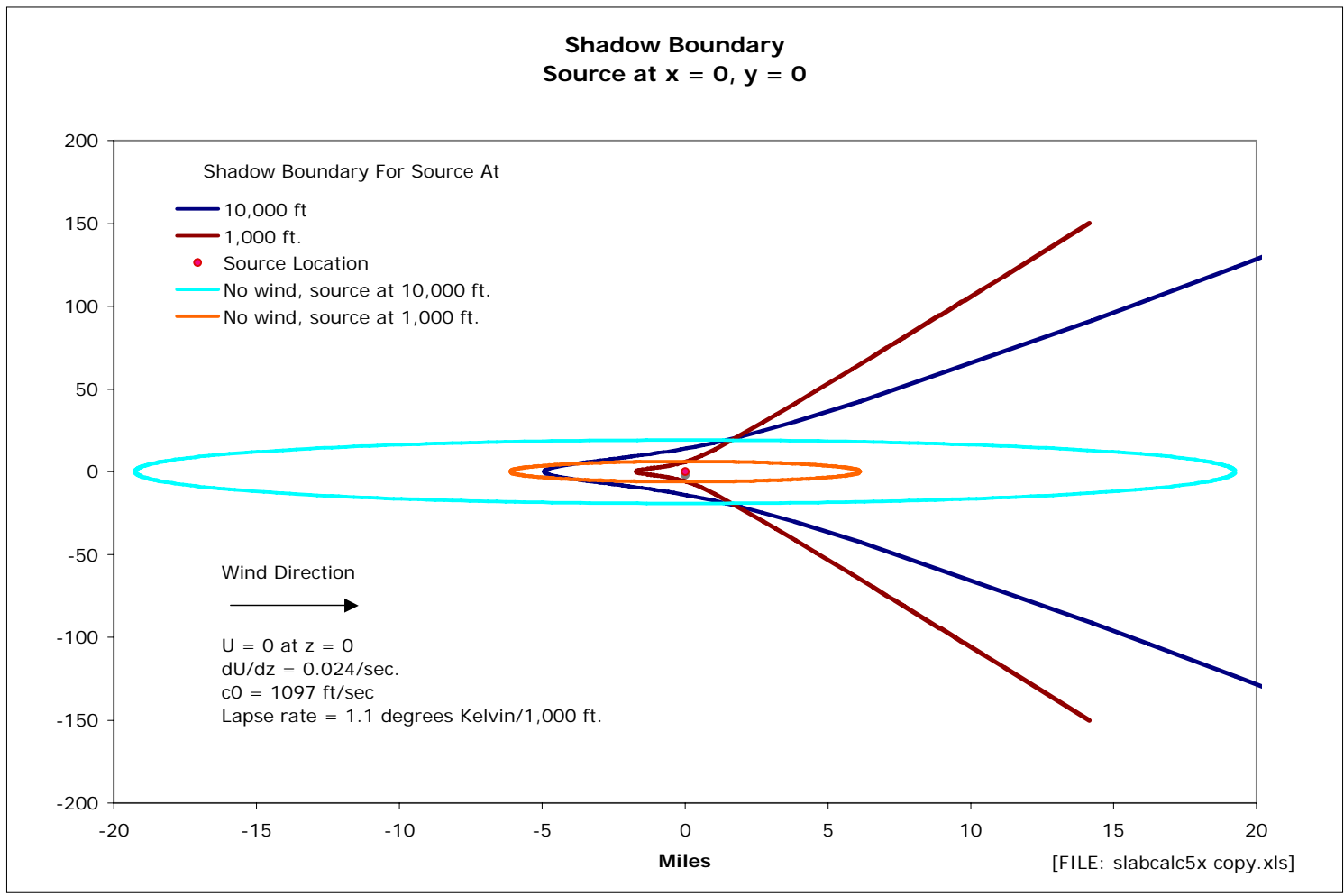

Figure 12. The shadow boundary for a point source in flow.

\section{Conclusions}

The characterization of the rays for a source in a stratified inhomogeneous moving medium and the connection of the behavior of each ray to its location in the $\left(\mathrm{s}_{\mathrm{x}}, \mathrm{s}_{\mathrm{y}}\right)$ plane provides for easy identification of those rays, and the associated point $\left(\mathrm{s}_{\mathrm{x}}, \mathrm{s}_{\mathrm{y}}\right)$, and allows the elimination of those rays that cannot contribute to the disturbance field at the ground before computer time is spent tracing them. Further, the identification of those rays that are members of the set of shadow forming rays, but that pass through a turning point before reaching the ground, makes it possible to easily and accurately determine the shadow boundary and thereby identify observers that cannot be connected to the source by any ray, thereby saving computer time.

\section{References}

${ }^{1}$ Lewis, R. M., and Keller, J. B.,"Asymptotic Methods for Partial Differential Equations: The Reduced Wave Equation and Maxwell's Equations," New York University Research Report EM-194, Jan. 1964.

${ }^{2}$ Blokhintsev, D. I., “Acoustics of a Nonhomogeneous Moving Medium,” NACA TM1399, 1946.

${ }^{3}$ Blokhintsev, D., "The Propagation of Sound in an Inhomogeneous and Moving Medium I," J. Acoust. Soc. Amer. Vol. 18, No. 2, 1946, pp. 322-328. 
${ }^{4}$ Pierce, Allan D., Acoustics, An Introduction to Its Physical Principles and Applications, McGraw-Hill, New York, 1981, Chapt. 8.

${ }^{5}$ Dowling, A. P., and Ffowcs Williams, J. E., Sound and Sources of Sound, Ellis Horwood,1983, Chapt. 5.

${ }^{6}$ Thompson, Robert J., "Ray Theory for an Inhomogeneous Moving Medium," J. Acoust. Soc. Amer. Vol. 51, No. 5 (2), 1971, pp. 1675-82.

${ }^{7}$ Thompson, Robert J., "Computing Meteorological Effects on Aircraft Noise," AIAA Journal Vol. 11, No. 1, 1973, pp. 121-23. 